Full Paper: The synthesis of double-hydrophilic block copolymers with a poly(ethylene glycol) block (PEG) and a block with pendant monophosphate ester groups based on a hydroxylated polybutadiene block (poly[2-(2hydroxy ethyl)ethylene] (PHEE) with variable degrees of phosphate substitution (up to $40 \%$ ) is described. It is shown that these block copolymers are very efficient scale inhibitors for $\mathrm{CaCO}_{3}$. The efficiency of these polymers is compared with block copolymers with an ionic block based on phosphorylated polyglycidol (PGL) with phosphorylation degrees up to $100 \%$. The phosphorylated polyglycidols were also used to modify the morphology of $\mathrm{CaCO}_{3}$ crystals. Instead of the typical rhombohedral calcite single crystals, superstructures of nanometer-sized particles are formed in the presence of these block copolymers when two different techniques were used: the fast double-jet technique and the slow Kitano-method. In the double-jet method, only spherical superstructures are obtained, whereas for the slower growth region covered by the Kitano method, complex cone-like or flower-like superstructures are formed.

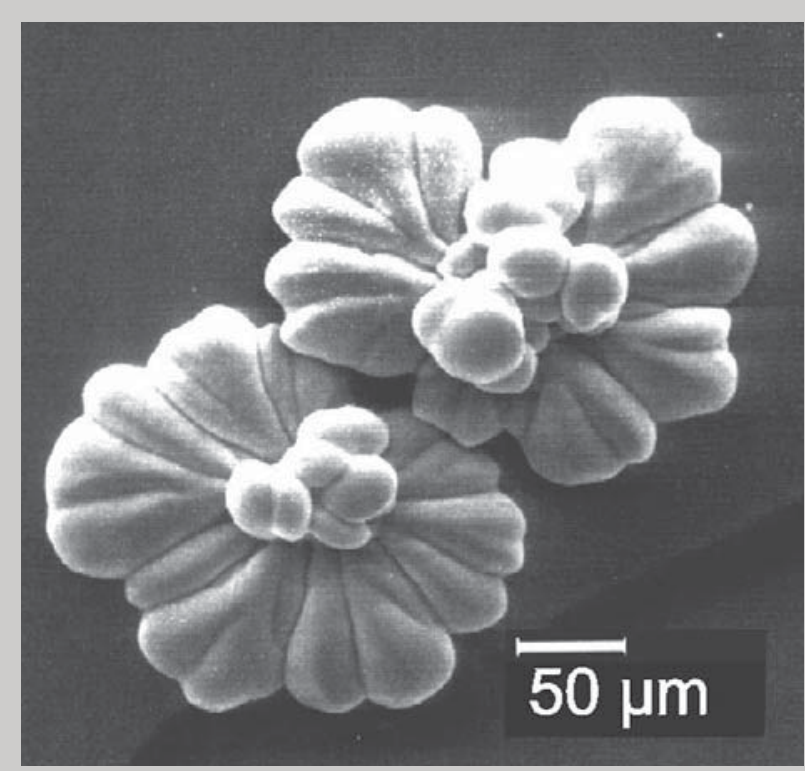

SEM micrographs of the formed $\mathrm{CaCO}_{3}$ structures taken after $80 \mathrm{~h}$ mineralization using the Kitano method at room temperature (Polymer PEG(45)- $b$-PGL(27) concentration $1 \mathrm{~g} \cdot \mathrm{l}^{-1}, 100 \%$ phosphorylation degree.

\title{
Double-Hydrophilic Block Copolymers with Monophosphate Ester Moieties as Crystal Growth Modifiers of $\mathrm{CaCO}_{3}$
}

\author{
Jan Rudloff, ${ }^{1}$ Markus Antonietti, ${ }^{1}$ Helmut Cölfen, ${ }^{* 1}$ Julia Pretula, ${ }^{2}$ Krzysztof Kaluzynski, ${ }^{2}$ Stanislaw Penczek ${ }^{2}$ \\ ${ }^{1}$ Max-Planck-Institute of Colloids and Interfaces, Colloid Chemistry, Research Campus Golm, \\ Am Mühlenberg, 14476 Golm, Germany \\ ${ }^{2}$ Center of Molecular and Macromolecular Studies Polish Academy of Sciences, Sienkiewicza 112, 90-363 Lodz, Poland
}

Keywords: anionic polymerization; $\mathrm{CaCO}_{3}$; diblock copolymers; double-hydrophilic block copolymers; nanocrystalline superstructures

\section{Introduction}

Functional, water-soluble polymers with the ability for ion binding play a major role in the inhibition of scale formation. ${ }^{[1,2]}$ These polymers are used in detergent formulations, but are also needed for applications such as oil drilling and for the protection of pipe systems. After the banning of polyphosphates as detergents for environmental reasons, polycarboxylates such as poly[(acrylic acid)co-(maleic acid)] or polyaspartate are most commonly used, however they do not perform as well as the polyphosphates.

Recently, a new class of block copolymers has been developed which shows superior scale-inhibition properties, namely the double-hydrophilic block copolymers. ${ }^{[3-7]}$ These block copolymers are composed of a short mineral binding block to interact with the formed crystals, and another hydrophilic moiety which weakly interacts with ions or crystal surfaces, thus promoting dissolution even 
under high-ionic-strength conditions. It was found that the mass efficiency of scale inhibition of $\mathrm{CaCO}_{3}$ was up to a factor of ten to fifteen times better than that of the standard commercial products. ${ }^{[4]}$

Besides the suppression of crystal precipitation, double-hydrophilic block copolymers are also able to modify the architecture of crystals in an exciting way. ${ }^{[7-12]}$ Depending on the selectivity of polymer adsorption on different crystal faces, spherical, needle-like, plateletlike, cigar-like or board-like crystals are nucleated, and undergo further alignment and aggregation to form complicated superstructures, such as fiber-bundles, dumbbells, hollow spheres, or neuron-like architectures. In the context of the present paper, it is important to mention that strongly binding polymers usually lead to almost spherical primary events, which often further aggregate to spherical superstructures.

In this paper, we describe the synthesis of a new class of double-hydrophilic block copolymers based on poly(ethylene glycol) (PEG) and poly[2-(2-hydroxy ethyl)ethylene] (PHEE) having monophosphate ester units on the side chain. To achieve this, we have used a phosphorylation process recently developed by three of the authors. ${ }^{[7]}$ The mineralization behavior of both classes of block copolymers is compared by the degree of phosphorylation and by the use of two different mineralization conditions, namely the double-jet technique and the Kitano procedure. ${ }^{[4,13]}$ The double-jet technique rapidly nucleates small crystals under stirred conditions, whereas the Kitano procedure allows structure building under slow growth and diffusion-controlled conditions.

\section{Experimental Part}

\section{Synthesis of Polymers}

All chemicals were of reagent grade and obtained from Aldrich. Ethylene oxide was obtained from Fluka. In the first step poly(ethylene glycol)-block-polybutadiene (PEG- $b$-PB) was synthesized by anionic polymerization as described in ref. ${ }^{[14]}$ The synthesized PEG- $b$-PB had the following characteristics: PB: $\bar{M}_{\mathrm{n}}=700 \mathrm{~g} \cdot \mathrm{mol}^{-1}(\mathrm{GPC}), \bar{M}_{\mathrm{w}} / \bar{M}_{\mathrm{n}}=1.11$ with 90\% 1,2-polymerization (NMR); PEG: $\bar{M}_{\mathrm{n}}=3700 \mathrm{~g} \cdot \mathrm{mol}^{-1}$ (NMR), PEG- $b$-PB: $\bar{M}_{\mathrm{n}}=4400 \mathrm{~g} \cdot \mathrm{mol}^{-1}, \bar{M}_{\mathrm{w}} / \bar{M}_{\mathrm{n}}=1.05$.

The PB block was then derivatized to PHEE by the introduction of hydroxyl functionalities via quantitative hydroboration with 9-bora-bicyclo[3.3.1]nonan (9-BBN) accord- ing to Ramakrishnan. ${ }^{[15]}$ The conversion of the vinylic side groups to hydroxyl groups was checked by ${ }^{1} \mathrm{H}$ NMR (disappearance of the signals of the double bond protons at 4.8$5.6 \mathrm{ppm}$ and appearance of the hydroxyl proton signal at $4.3 \mathrm{ppm})$.

The PEG- $b$-PHEE can be phosphorylated by various methods. In this work, three different approaches were applied with varying success:

a) Enzymatic phosphorylation by phosphorylase kinase (enzyme commission code EC 2.7.1.38) in the presence of 1.3 equivalents of adenosine triphosphate (ATP) (according to $-\mathrm{OH}$-groups) in a $1-\mathrm{mg} \cdot \mathrm{ml}^{-1}$ block copolymer solution of aqueous phosphate buffer at $\mathrm{pH} 7.7$ by stirring the solution overnight at $30^{\circ} \mathrm{C}$ proved unsatisfactory, with phosphorylation degrees not higher than 5$10 \%$ being obtained. ${ }^{[16]}$

b) Phosphorylation according to Sikes, ${ }^{[17]}$ which was introduced for the phosphorylation of peptides, also led to rather low phosphorylation degrees of $20 \%$. The phosphorylating agent was $\mathrm{POCl}_{3}{ }^{[17]}$ Partial hydrolysis of $\mathrm{POCl}_{3}$ in the first reaction step by 2 equivalents of water led to a product with one active chlorine atom. The block copolymer was subsequently added, followed by stirring of the solution overnight at ambient temperature.

c) Phosphorylation according to the procedure developed by three of the authors allowed degrees of phosphorylation of the $-\mathrm{OH}$ groups of up to $40 \% .{ }^{[7]}$

The raw products obtained were cleaned by exhaustive ultrafiltration in pure water using Millipore ${ }^{\mathrm{TM}}$ membranes with a MWCO (molecular weight cut-off) of $1000 \mathrm{~g} \cdot \mathrm{mol}^{-1}$. After freeze-drying of the aqueous polymer solutions, the degree of phosphorylation was determined with quantitative ${ }^{31} \mathrm{P}$ NMR spectroscopy, by comparing the signals of an internal standard (tert-butyl phosphonic acid at $30 \mathrm{ppm}$ ) with the signal of the phosphorylated product $(4.65 \mathrm{ppm})$ in $\mathrm{D}_{2} \mathrm{O}$ (at pD12). Virtually quantitative yields of polymers were obtained after purification.

In addition, double-hydrophilic block copolymers based on PEG and phosphorylated polyglycidol (PGL) were examined in order to make a comparison with the block copolymers described above. The synthesis of this second class of double-hydrophilic block copolymers is described elsewhere. ${ }^{[7]}$ Thus, according to ref. ${ }^{[7]}$ the $-\mathrm{OH}$ end groups of poly(ethylene glycol) monomethyl ether were converted into the alkoxy anions in order to obtain a macroinitiator for the anionic polymerization of the glycidol. The $-\mathrm{OH}$ group of the glycidol was initially protected with methyl vinyl ether. The obtained protected PEG- $b$-PGL block copolymer was deprotected under acidic conditions and then phosphorylated with $\mathrm{POCl}_{3}$ dissolved in triethylphosphate. ${ }^{[7]}$ The polymers

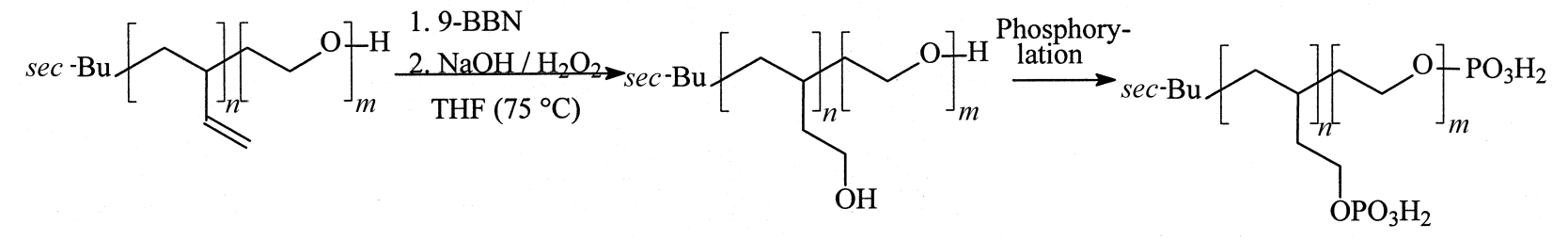

Scheme 1. Hydroboration of PEG- $b$-PB to PEG- $b$-PHEE and subsequent phosphorylation. 
Table 1. Molecular parameters of the phosphorylated block copolymers; the numbers in parentheses represent the repeating units in the polymer block segments.

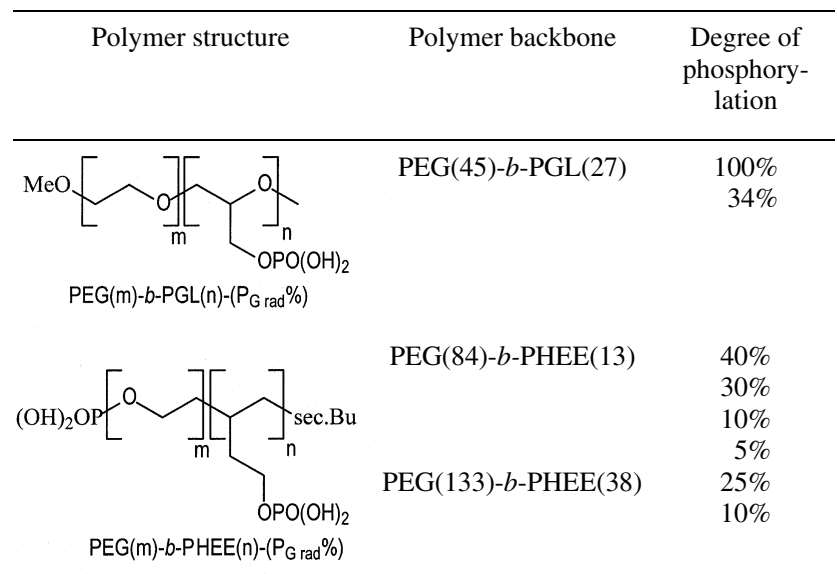

were exhaustively ultrafiltrated in pure water using Millipore $^{\mathrm{TM}}$ membranes with an MWCO of $1000 \mathrm{~g} \cdot \mathrm{mol}^{-1}$ before further use in mineralization experiments.

Table 1 summarises the molecular parameters of the polymers employed in this examination.

\section{Mineralization Experiments}

Scale-inhibition efficiencies were determined in a double-jet set-up described earlier. ${ }^{[4]}$ The set-up consists of a thermostated vessel $\left(25^{\circ} \mathrm{C}\right)$ containing a $100-\mathrm{ml}$ solution of the block copolymer in varying concentrations $\left(0.1-2.0 \mathrm{~g} \cdot \mathrm{l}^{-1}\right)$, and adjusted to $\mathrm{pH}=8.5$. The solution is kept under argon to prevent dissolution of atmospheric $\mathrm{CO}_{2}$. The two reactants, $0.5-\mathrm{M} \mathrm{Na} \mathrm{CO}_{3}$ in $\mathrm{H}_{2} \mathrm{O}(\mathrm{pH}=11.57)$ and $0.5-\mathrm{M} \mathrm{CaCl} \mathrm{Can}_{2}$ in $\mathrm{H}_{2} \mathrm{O}$ (brought to $\mathrm{pH}=8.5$ ), are injected via two capillaries which are joined together at their ends under vigorous stirring to avoid heterogeneous nucleation. The injection speed was 0.5 or $2.5 \mathrm{ml} \cdot \mathrm{h}^{-1}$ so that the formation rate of $\mathrm{CaCO}_{3}$ is 0.25 or $1.25 \mathrm{~mol} \cdot \mathrm{h}^{-1}$. The double-jet technique provides high supersaturation and thus, immediate nucleation of $\mathrm{CaCO}_{3}$ at the mixing spot, followed by immediate particle transport to regions of lower $\mathrm{CaCO}_{3}$ concentration which are in the proximity of the modifying block copolymers. Initially formed crystal nuclei will dissolve as long as the overall concentration is below the saturation concentration, whereas particles formed later are stabilized by the polymer. The onset of macroscopic precipitation of $\mathrm{CaCO}_{3}$ that occurs after excess addition is easily detected by a sudden increase in turbidity. This onset time was recorded and is taken as a measure for the capacity of each polymer for scale inhibition.

In contrast, the Kitano technique runs on a longer time scale in order to exclude kinetic effects in the precipitation experiment. ${ }^{[13]}$ A supersaturated solution of $\mathrm{CaCO}_{3}$ is prepared by bubbling $\mathrm{CO}_{2}$ gas through a slurry of $5 \mathrm{~g}$ of $\mathrm{CaCO}_{3}$ in 41 of pure water for $60 \mathrm{~min}$. The $\mathrm{CaCO}_{3}$ was subsequently filtered off and $\mathrm{CO}_{2}$ was then bubbled through for another $30 \mathrm{~min}$ in order to dissolve the remaining $\mathrm{CaCO}_{3}$ particles. The equilibrium of $\mathrm{CaCO}_{3} / \mathrm{HCO}_{3}^{-}$is shifted to $\mathrm{HCO}_{3}^{-}$according to Scheme 2. $\mathrm{CaHCO}_{3}$ is more soluble in water than

$$
\mathrm{CaCO}_{3(\mathrm{~s})}+\mathrm{CO}_{2(\mathrm{~g})}+\mathrm{H}_{2} \mathrm{O}_{(\mathrm{l})} \rightleftharpoons \mathrm{Ca}_{(\mathrm{aq})}^{2+}+2 \mathrm{HCO}_{3(\mathrm{aq})}^{-}
$$

Scheme 2. The $\mathrm{CaCO}_{3} / \mathrm{HCO}_{3}^{-}$equilibrium.

$\mathrm{CaCO}_{3}$, therefore the equilibrium in Scheme 2 shifts to the right, leading to a higher concentration of $\mathrm{Ca}^{2+}$ in the aqueous phase.

The solution was left in unsealed vessels. Carbon dioxide is formed on a time scale of days. Hence the equilibrium shifts back to the left, generating a $\mathrm{CaCO}_{3}$ supersaturation in the aqueous phase. Precipitation of $\mathrm{CaCO}_{3}$ begins at the air/ water interface, where the lowest concentration of $\mathrm{CO}_{2}$ is found.

\section{Instrumental Techniques}

Scanning electron microscopy (SEM) was performed on a DSM 940A (Carl Zeiss, Jena) and images were taken with a digital camera connected to the SEM apparatus. The crystals were taken directly from the solution. Most of the solution was soaked off the sample stub using tissue paper in order to minimize additional crystallization due to drying on the SEM stub. To prove that the SEM sample preparation does not alter the $\mathrm{CaCO}_{3}$ crystals, optical microscopy images were taken in solution at the same time with an Olympus BX50 microscope.

\section{Results and Discussion}

\section{Polymer Synthesis}

The synthesis of the PHEE via anionic polymerisation of butadiene and ethylene oxide according to Förster and Krämer and the following hydroboration after the method of Ramakrishnan worked with virtually quantitative yields and complete conversion of the functional groups. ${ }^{[14,15]}$ The enzymatic phosphorylation of the $-\mathrm{OH}$ side groups by phosphorylase kinase was unsuccessful because this enzyme is designed to phosphorylate a specific serine residue in a protein superstructure. ${ }^{[16]}$ The regular $-\mathrm{OH}$ pattern along the PHEE block is not sufficient to mimic the target structure of the enzyme according to the "lock and key principle", which is the reason for the high selectivity of enzymes.

On the other hand the phosphorylation according to the method of Sikes suffered from a number of by-products mainly caused by the less than stoichiometric hydrolysis of $\mathrm{POCl}_{3}$ and subsequent oligomerization, crosslinking, etc. - decreasing the phosphorylation degree of PHEE to only $20 \%$, and resulting in a large amount of impurities. $^{[17]}$

Finally, the phosphorylation following the method of Penczek et al. gave phosphorylation degrees of up to $40 \% .{ }^{[7]}$ A complete conversion of all $-\mathrm{OH}$ groups into phosphate groups, which occurs in the case of the PEG- $b$ PGL block copolymers could not be achieved. Apparently, the steric requirement of a phosphate group is too demanding for the PEG- $b$-PHEE backbone. The distance 
between two $-\mathrm{OH}$ groups is larger by one oxygen/monomer unit in the PEG- $b$-PGL backbone than in the PEG- $b$ PHEE backbone (monomer unit PHEE $=0.26 \mathrm{~nm}$, PGL = $0.36 \mathrm{~nm}$ compared to phosphate $=0.22 \mathrm{~nm}$ ). Therefore, complete phosphorylation in the case of PEG- $b$-PGL was possible despite the fact that PGL has only one $-\mathrm{CH}_{2}$ group in its side chain compared with the two $-\mathrm{CH}_{2}$ groups in side chain of the phosphorylated PHEE, which suggests that the former has less side-chain flexibility.

Moreover, the PHEE backbone is much more hydrophobic than the PGL backbone, having one oxygen atom less than the PGL polymer backbone. Therefore in a polar reaction medium, such as triethylphosphate which was used as solvent in the phosphorylation reaction, the PHEE backbones may aggregate making only a part of the $-\mathrm{OH}$ side groups available for phosphorylation.

\section{Crystallization Inhibition Efficiency}

In the first set of experiments, the crystallization-inhibition efficiencies were determined for the different phosphorylated block copolymers for $\mathrm{CaCO}_{3}$. This is expressed as the mass ratio $s_{\mathrm{m}}$, which defines the amount of polymer required to keep $1 \mathrm{~g}$ of $\mathrm{CaCO}_{3}$ colloidally stable in solution (Equation 1). The lower the $s_{\mathrm{m}}$ value, the better the efficiency of a given polymer. ${ }^{[4]}$

$$
s_{\mathrm{m}}=\frac{m_{\text {Polymer }}}{m_{\mathrm{CaCO}_{3}}}
$$

In order to gain a better idea about the crystallizationinhibition efficiency on the basis of each active functional group, the $s_{\mathrm{f}}$ value is introduced, which describes the stabilization efficiency according to the number of functional groups of the block copolymer (Equation 2).

$$
s_{\mathrm{f}}=\frac{n_{\mathrm{Polymer}} \cdot x}{n_{\mathrm{CaCO}_{3}}}
$$

where $n$ is the quantity in moles and $x$ is the number of functional groups per polymer molecule on a number basis.

The mass of polymer in the solution is known, whereas the mass of $\mathrm{CaCO}_{3}$ can be calculated from the reactant addition rate and the turbidity onset time in the double-jet precipitation experiment. The results for the different polymers are summarized in Table 2.

From Table 2, it can be seen that the phosphorylated PEG- $b$-PGL shows very high crystallization-inhibition efficiencies of $s_{\mathrm{m}}=0.8-1.6$, values comparable to the best values of $s_{\mathrm{m}}=1.6-3.1$ reported for PEG- $b$-PEDTA 3 in ref. ${ }^{[4]}$ Also, the phosphorylated PEG- $b$-PHEE show high efficiencies of 1.4-4.8 which are better than the values of 8-23 found for PEG- $b$-EDTA. ${ }^{[4]}$

Commercial builders for water treatment with $s_{\mathrm{m}}=2.6$ and 2.1 for polyaspartate (Pasp) and poly[(acrylic acid)-
Table 2. Crystallization inhibition efficiency for a polymer concentration of $1 \mathrm{~g} \cdot \mathrm{l}^{-1}$ in a volume of $100 \mathrm{ml}$.

\begin{tabular}{ccccc}
\hline Polymer backbone & $\begin{array}{c}\text { Degree of } \\
\text { phosphory- } \\
\text { lation }\end{array}$ & \begin{tabular}{c} 
Flow rate \\
\cline { 5 - 5 }
\end{tabular} & $s_{\mathrm{m}}$ & $s_{\mathrm{f}}$ \\
& & & & \\
\hline PEG(84)- $b$-PHEE(13) & $40 \%$ & 0.5 & 3.8 & 0.37 \\
& $30 \%$ & 0.5 & 4.3 & 0.38 \\
& & 2.5 & 4.8 & 0.37 \\
& $10 \%$ & 0.5 & 4.5 & 0.11 \\
& & 2.5 & 3.0 & 0.08 \\
& & 0.5 & 2.6 & 0.07 \\
PEG(133)- $b$-PHEE(38) & $25 \%$ & 0.5 & 1.4 & 0.04 \\
& & 2.5 & 3.0 & 0.35 \\
& $10 \%$ & 0.5 & 4.4 & 0.34 \\
PEG(45)- $b$-PGL(27) & $100 \%$ & 2.5 & 4.1 & 0.17 \\
& & 0.5 & 1.6 & 0.59 \\
& & 2.5 & 1.6 & 0.59 \\
Polyaspartate & & 0.5 & 0.8 & 0.14 \\
Sokalan ${ }^{\text {TM }}$ & & 2.5 & 1.2 & 0.21 \\
& & 2.5 & 1.6 & 1.20 \\
& & 2.5 & 1.1 & 1.29
\end{tabular}

co-(maleic anhydride)] (Sokalan $\left.{ }^{\mathrm{TM}}\right)$, respectively, were investigated in parallel experiments, and showed crystallization inhibition efficiencies in the same range of the block copolymers on a mass basis. However, with respect to the functional groups per aliquot of stabilized mineral, the double-hydrophilic block copolymers are clearly more efficient than the commercial builders which is reflected in the $s_{\mathrm{f}}$ values (see Table 2). Compared to the commercial builders Pasp and Sokalan ${ }^{\mathrm{TM}}$, the efficiency per functional group is higher by a factor of 2-7. A surprising systematic trend can be deduced from the $s_{\mathrm{f}}$ values versus the phosphorylation degree for all investigated block copolymers: The higher the phosphorylation degree, the lower the stabilization efficiency per functional group, implying that not all phosphate groups are needed as sticker groups for $\mathrm{CaCO}_{3}$.

It must be stated that the $s_{\mathrm{m}}$ values are calculated from visual observations of the first occurrence of turbidity so that reproducible, exact values are hard to obtain. Therefore, $s_{\mathrm{m}}$ is expressed as a range rather than as a single value. Also, experiments using other $\mathrm{CaCO}_{3}$ addition rates, sample volumes or polymer concentrations indicate (data not shown) that the $\mathrm{CaCO}_{3}$ addition rate and the sample volume are of minor importance, however the overall concentration of polymer and $\mathrm{CaCO}_{3}$ is important. Under constant conditions, the crystallization inhibition efficiency tends to increase with decreasing polymer concentration, as has been observed for polymer concentrations the range of $0.1-4 \mathrm{~g} \cdot \mathrm{l}^{-1}$. This is in good agreement with general considerations concerning the colloidal stability of the formed polymer/crystal pre-structures. As a consequence, although a polymer concentration of $1 \mathrm{~g} \cdot \mathrm{l}^{-1}$ was used as a standard in the tests for the crystal- 
lization-inhibition efficiency, a lower polymer concentration of $0.1-0.5 \mathrm{~g} \cdot \mathrm{1}^{-1}(\approx 100-500 \mathrm{ppm})$ can also be applied for equally efficient scale inhibition resulting in lower $s_{\mathrm{m}}$ values. For the example of PEG(45)- $b$ PGL(27)(100\%), we obtained $s_{\mathrm{m}}=0.96\left(\mathrm{c}=0.5 \mathrm{~g} \cdot \mathrm{l}^{-1}\right)$, $s_{\mathrm{m}}=1.94\left(\mathrm{c}=1 \mathrm{~g} \cdot \mathrm{l}^{-1}\right)$ and $s_{\mathrm{m}}=2.13\left(\mathrm{c}=2 \mathrm{~g} \cdot \mathrm{l}^{-1}\right)$.

Surprisingly the $s_{\mathrm{m}}$ values also slightly depend on the reactant addition rate for phosphorylated PEG- $b$-PHEE. At higher flow rates $\left(2.5 \mathrm{ml} \cdot \mathrm{h}^{-1}\right.$ vs. $\left.0.5 \mathrm{ml} \cdot \mathrm{h}^{-1}\right)$ higher stabilization capacities were observed. This may be explained by kinetic effects, such as the time of nucleation and particle growth which allows the polymer more time to interact with larger structures than for faster addition rates.

\section{$\mathrm{CaCO}_{3}$ Morphology and Crystal Modification}

As expected for double-hydrophilic block copolymers with strong and non-selective surface binding, the obtained structures are predominantly composed of vaterite nanocrystallites initially stabilized by the copolymers. Using the fast double-jet technique with stirring, these primary nanocrystallites aggregate further to form spherical superstructures at higher concentrations as a consequence of the short stabilizing block. A typical event of the double-jet mineralization with phosphorylated polymers leading to well-defined superstructures of $\mathrm{CaCO}_{3}$ nanoparticles is shown in Figure 1.

Wide-angle X-ray analysis (WAXS) reveals that the 10-20- $\mu \mathrm{m}$-sized spheres consist of 20-40-nm-sized nanocrystals, depending on the phosphorylation degree of the copolymer and the speed of $\mathrm{CaCO}_{3}$ addition. For the mineralization with the phosphorylated PEG- $b$-PHEE block copolymers the $\mathrm{CaCO}_{3}$ modification vaterite was obtained in all cases, independent of the degree of phosphorylation and flow rate. In the case of the phosphorylated PEG- $b$-PGL a dependence of the crystal modification on the flow rate was observed. At high-jetting flow rates $\left(2.5 \mathrm{ml} \cdot \mathrm{h}^{-1}\right)$ only vaterite was formed, whereas at lower flow rates a mixture of vaterite and calcite was formed. This reflects an insufficient stabilization of the kinetically favoured modification vaterite by the polymer, which then partly transforms into the thermodynamically stable calcite at slower $\mathrm{CaCO}_{3}$ addition rates.

Overall, these results are in good agreement with previously described experiments on partly phosphorylated PEO- $b$-PMAA's, where micron sized spheres with low polydispersity were found to be composed of 9-12-nmsized vaterite and calcite nanocrystals. ${ }^{[5]}$

\section{$\mathrm{CaCO}_{3}$ Crystallization by the Kitano Method}

Since non- or weakly- selective binding and rapid precipitation results in trivial spherical superstructures, more selective growth conditions have to be applied, in order

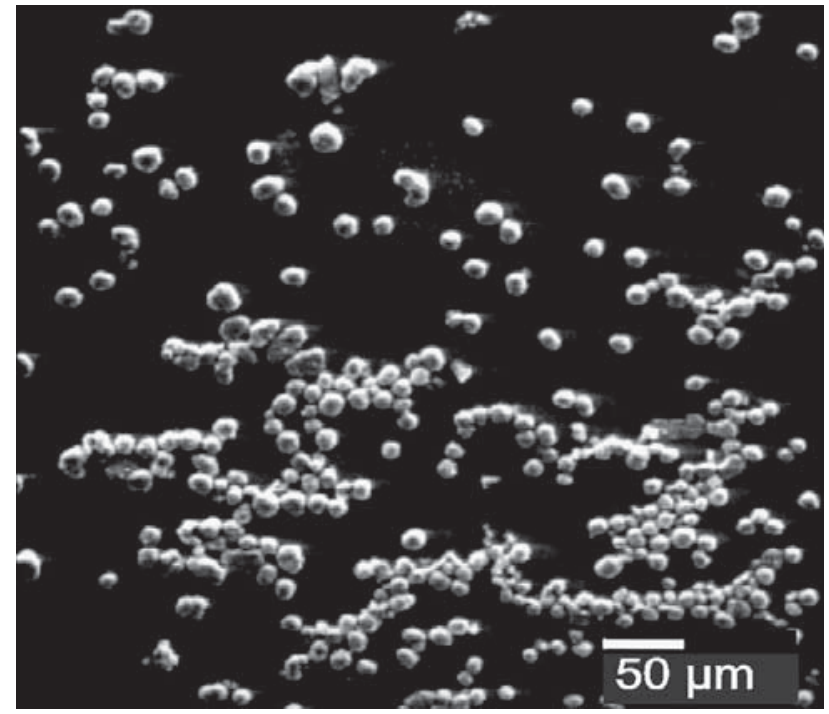

Figure 1. SEM micrograph with typical results of a double-jet mineralization with $100 \%$ phosphorylated PEG(45)- $b$-PGL(27) $\left(c=2 \mathrm{~g} \cdot \mathrm{l}^{-1}\right.$, flow rate $2.5 \mathrm{ml} \cdot \mathrm{h}^{-1}$, after $\left.75 \mathrm{~min}\right)$.

to be sensitive towards the minor differences between the polymers. This is done by the Kitano technique. For a stirred Kitano solution with a relatively fast $\mathrm{CO}_{2}$ evaporation, spherical superstructures were still obtained - an analogous result to the double-jet experiments.

When the Kitano mixture is not stirred, the structures can develop on the time scale of days, and new shapes appear. The particles are nucleated in the upper layers near the $\mathrm{CO}_{2}$ bubbles at the solution interface by heterogeneous nucleation, because there is a local maximum in $\mathrm{CaCO}_{3}$ supersaturation caused by the low carbon dioxide concentration (according to Scheme 2), and the mineral grows at the water/air interface. Some typical examples obtained for the series of the glycidol polymers are shown in Figure 2.

For the system with lower degrees of phosphorylation, a typical "fried-egg" morphology develops at the air/water interface (Figure 2e,f). Looking at the time development of these structures, it is seen that a ring-like morphology of $\mathrm{CaCO}_{3}$ is formed first, presumably templated by a $\mathrm{CO}_{2}$ bubble (Figure 3 ). In the next step, the rings grow to hollow half spheres which are also templated by the structure of carbon dioxide bubbles. Beginning from the core, the outer brim grows around the structure. WAXS diffraction experiments show that these materials consist of vaterite nanocrystals with sizes between 10 and $15 \mathrm{~nm}$.

This growth mechanism is analogous to the vaterite microsponges formed by the same method but under different solution conditions, in that case a microemulsion where the $\mathrm{CO}_{2}$ bubbles template microcavities in the crystallizing $\mathrm{CaCO}_{3}$. However in our case, the centre of the "fried-egg" morphology is slightly bigger. ${ }^{[18]}$

Increasing the degree of phosphorylation from polymer PEG(45)- $b$-PGL(27) to $100 \%$ of all hydroxy groups 
a)

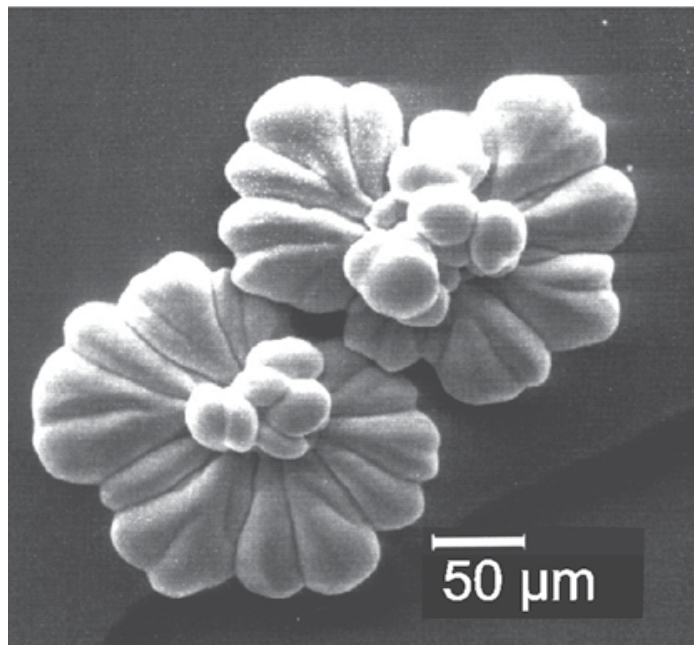

c)

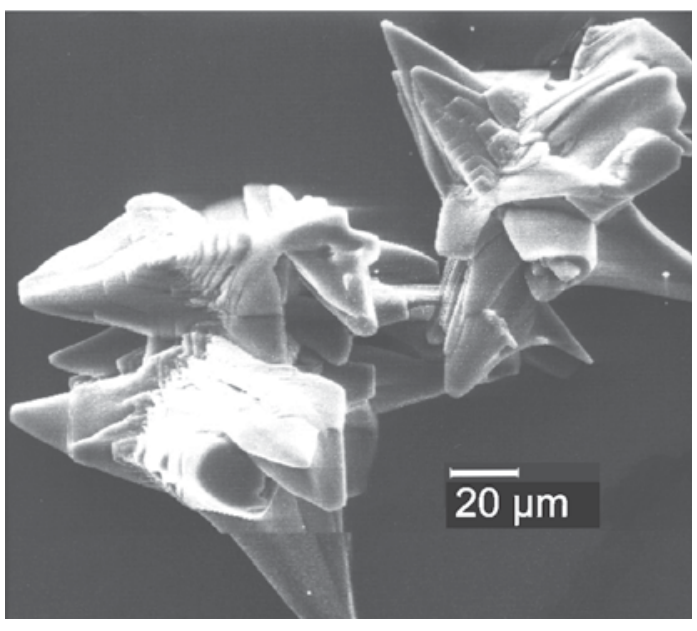

e)

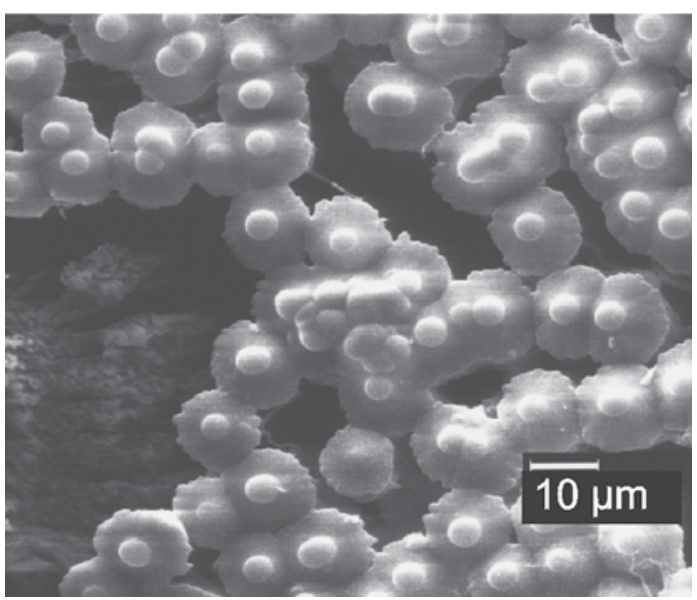

b)

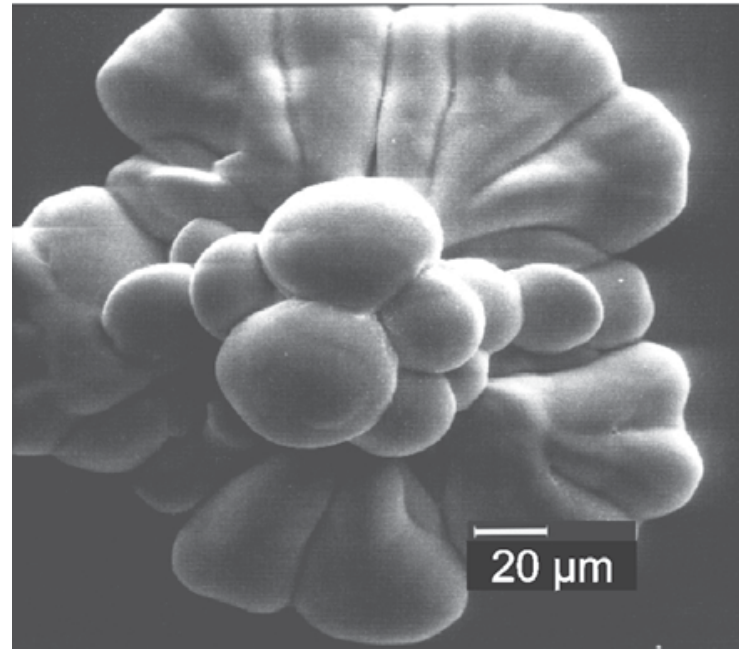

d)

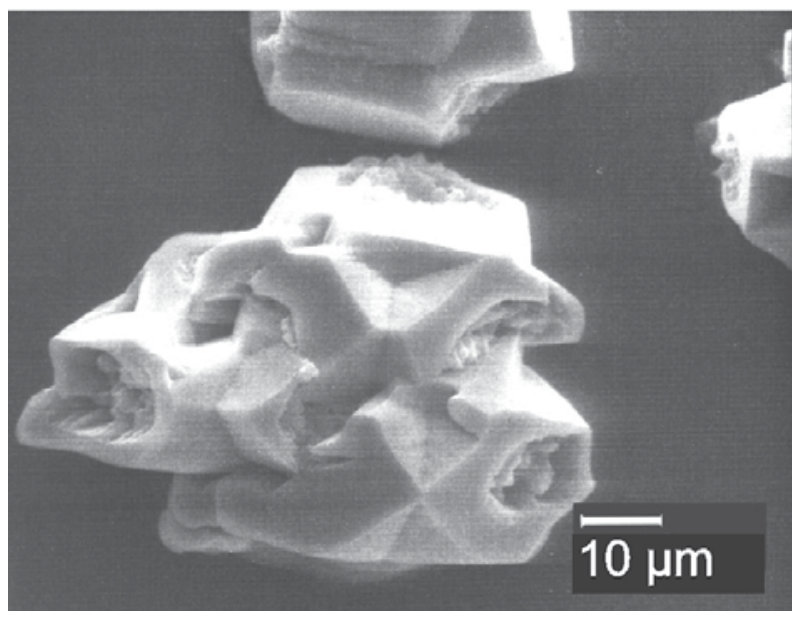

f)

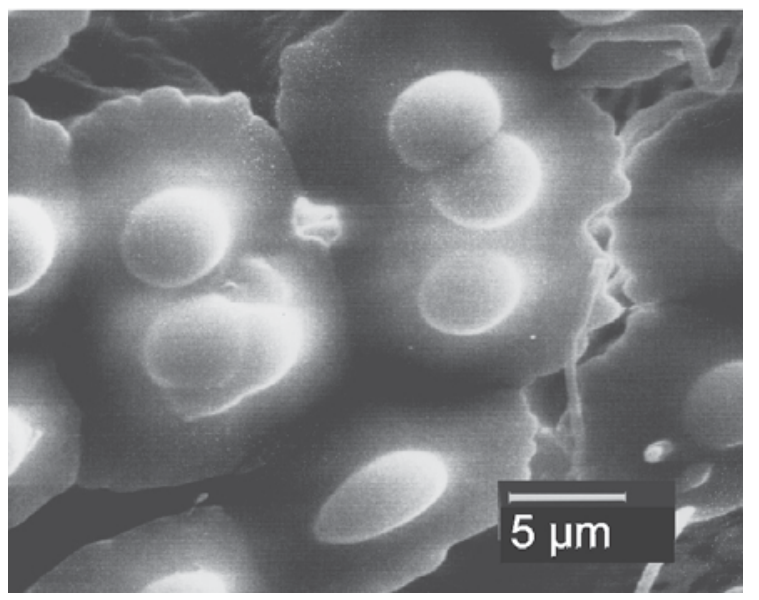

Figure 2. SEM micrographs of the formed $\mathrm{CaCO}_{3}$ structures taken after $80 \mathrm{~h}$ mineralization time with the Kitano method at room temperature (Polymer PEG(45)- $b$-PGL(27) concentration $1 \mathrm{~g} \cdot \mathrm{l}^{-1}$ ): (a-d) Polymer with $100 \%$ phosphorylation degree; $(\mathrm{e}, \mathrm{f})$ with $34 \%$. 


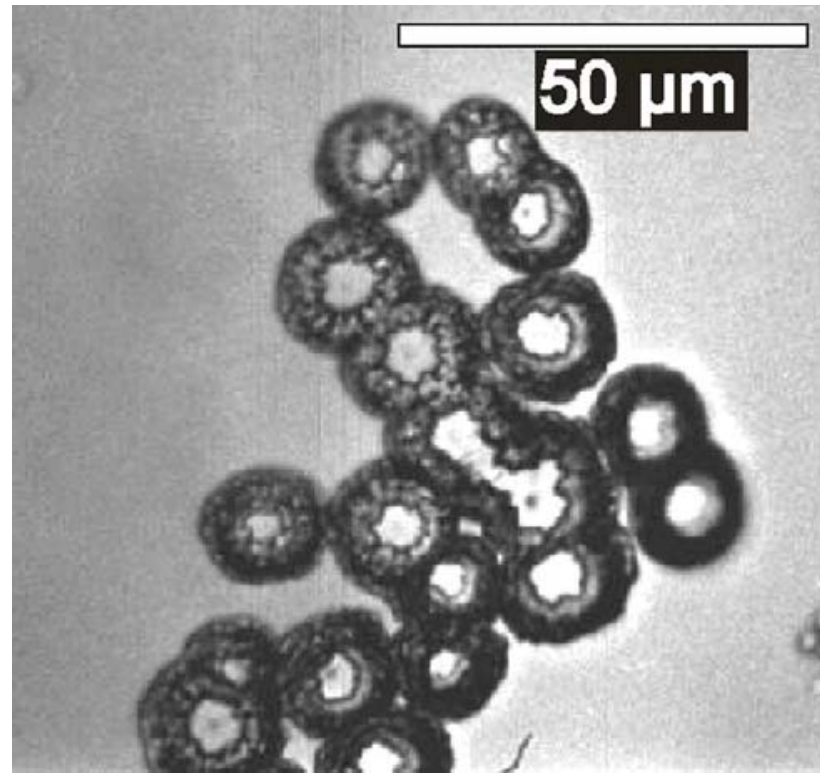

Figure 3. Light micrograph from the first stage of the growth process of $\mathrm{CaCO}_{3}$ in the presence of $1 \mathrm{~g} \cdot \mathrm{l}^{-1} 34 \%$ phosphorylated PEG(45)- $b$-PGL(27), showing the ring morphology of $\mathrm{CaCO}_{3}$.

results in related $\mathrm{CaCO}_{3}$ structures, also formed at the air water interface, as evidenced from the flat surface at one end, as partially observed in the SEM micrographs. These are flower-like superstructures, where the center is formed by more than a single spherical primary aggregate (the "pollen"), and the "brim" is also separated into a number of curved petals (Figure $2 \mathrm{a}, \mathrm{b}$ ). Increasing the degree of phosphorylation obviously leads to a trend towards hybrid structures with higher curvature. These structures were formed in a range of polymer concentrations between 0.2 and $2 \mathrm{~g} \cdot \mathrm{l}^{-1}$, and only at concentrations as low as $0.1 \mathrm{~g} \cdot \mathrm{1}^{-1}$ was the polymer concentration not sufficient to stabilize structured particles, resulting in spherical and irregular crystals.

The growth mechanism of these superstructures is slightly different from those obtained in presence of the lower phosphorylated block copolymer. Light microscopy of the early growth stages indicates an aggregation of several spheres to a core, followed by dendritic growth of the outer brim (data not shown). The initially dendritic brim is overgrown at a later stage, as revealed by optcal microscopy in solution. WAXS reveals that the dried mixture of the formed particles consisting of $18-\mathrm{nm}$ vaterite nanocrystallites and 29-nm calcite crystals.

In the case of the $100 \%$ phosphorylated PEG(45)- $b$ PGL(27) block copolymer, a second species consisting of calcite is formed in the continuous solution, in the form of irregular crystals (Figure $2 \mathrm{c}, \mathrm{d}$ ). In contrast to the flower-like species, crystal faces are exposed which is indicative that these crystals are not superstructures, but at least partly single crystals. We assume that these species are presumably nucleated from fragmented parts of
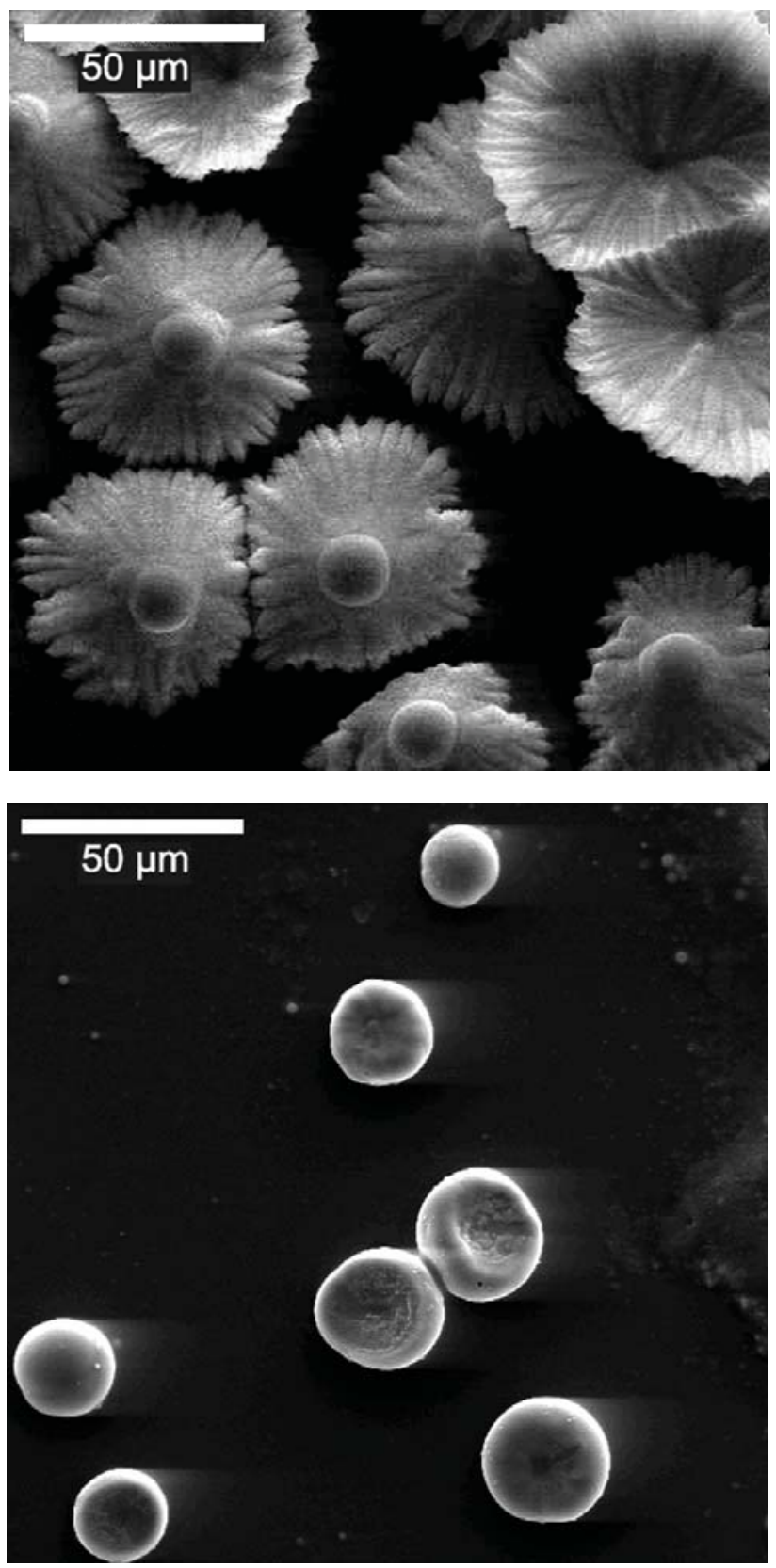

Figure 4. SEM micrographs of the formed $\mathrm{CaCO}_{3}$ structures formed under the influence of Polymer PEG(84)- $b$-PHEE(13): (a) with $40 \%$ phosphorylation degree; (b) with $10 \%$. (The pictures were taken after $80 \mathrm{~h}$ mineralization using the Kitano method at room temperature, with a polymer concentration of $\left.1 \mathrm{~g} \cdot 1^{-1}\right)$ :

the "pollen", since these crystals are not found in the experiments with the lower phosphorylated polymer. Although made of calcite, as shown by WAXS measurements, it is seen that the crystals exhibit rather unusual angles. This means that the polymer doesn't absorb to the usual exposed calcite surfaces - although no regularity is observable.

It is emphasised that both of the phosphorylated PEG(45)- $b$-PGL(27) polymers show a molecular compo- 
sition with a rather large crystal binding block, which brings into doubt the reversibility of polymer adsorption. Polymers with shorter blocks of this type were not examined for the mineralization experiments. This is the reason why PEG- $b$-PHEE block copolymers were synthesized with a smaller crystal binding block.

Using these polymers with different phosphorylation degrees, a rich variety of crystal morphologies was obtained which will be reported in a forthcoming study. Figure 4 shows some typical examples of different mineral morphologies formed under the influence of phosphorylated PEG- $b$-PHEE block copolymers.

\section{Conclusion}

A set of double-hydrophilic block copolymers with a hydrophilic PEG block and a polyanionic phosphate functionalized block were synthesized based on hydroxylated PEG- $b$-PHEE. Well defined double-hydrophilic block copolymers with a narrow molar mass distribution were obtained from anionic polymerization. A novel phosphorylation reaction developed for the quantitative phosphorylation of PGL blocks was also found to phosphorylate the PHEE block in the expected manner. Therefore, different desired phosphorylation degrees of up to $40 \%$ could be realized with this reaction in contrast to lower phosphorylation degrees via enzymatic phosphorylation or a phosphorylation procedure for the phosphorylation of peptides. The degree of phosphorylation of the PEG- $b$ PHEE block copolymers was limited to $40 \%$, possibly due to the steric congestion.

The phosphorylated PEG- $b$-PHEE with different phosphorylation degrees as well as PEG- $b$-PGL were applied as an additive for $\mathrm{CaCO}_{3}$ crystallization and showed a very good crystal-inhibition efficiency for $\mathrm{CaCO}_{3}$, comparable with that of commercial builders for water treatment, but with a higher efficiency on basis of the number of functional groups. This result emphasizes that the block-copolymer structure with the separation of stabilizing and sticking moiety leads to a better stabilization efficiency.

Beside a good stabilization efficiency for $\mathrm{CaCO}_{3}$, the block copolymers also showed a significant influence on the particle morphology as revealed for the phosphorylated PEG- $b$-PGL. Fast crystallization of nanocrystals by the double-jet method yielded spherical particles, which were superstructures of vaterite/calcite nanocrystals. A size of around $20 \mathrm{~nm}$ was obtained, independent of the polymer phosphorylation degree. This is in agreement with the result of another only partially phosphonated block copolymer. ${ }^{[5]}$

This indicates that both acidic phosphonate or phosphate groups are very good sticker groups for $\mathrm{CaCO}_{3}$ which directly adsorb once a nanoparticle is formed and thus lead to nanoparticle stabilization. At a later stage and higher particle concentration, aggregation of the primary formed nanoparticles into the spherical superstructures occurs. The exact mechanism for this is not fully explored, but is certainly due to insufficient colloidal stabilization by the small, sterically stabilizing PEG unit. The resulting relatively weak aggregation allows for rearrangements between the building blocks, and a spherical shape of the superstructure results from the surface minimization.

Slow crystallization by the Kitano method yields remarkably different morphologies, because here, the $\mathrm{CaCO}_{3}$ nucleation occurs slowly at the air/water interface. Therefore smaller differences in the polymer crystal interaction energy landscape as well as diffusional effects become more important. Although superstructures were formed from 10-30-nm vaterite and calcite nanoparticles, analogous to the fast double-jet precipitation, the particle morphology was found to be much different, exhibiting a higher level of superstructure formation of the nanocrystalline building units and depending on the degree of phosphorylation of the polymer. These results indicate that phosphorylated block copolymers are promising additives for the formation of superstructures composed of nanometer-sized primary $\mathrm{CaCO}_{3}$ particles.

Acknowledgement: This work was partially supported by the Polish State Committee for Scientific Research (KBN) grant 7 T09A 156 21. We also thank the Max-Planck Society and DFG (SFB 448) for financial support. BASF $A G$, Ludwigshafen is acknowledged for leaving polyaspartate and Sokalan ${ }^{\mathrm{TM}}$ at our disposal. We thank Claudia Günther for supporting the mineralization experiments. Erich $C$. is acknowledged for inspiring discussions.

[1] Z. Amjad, J. Colloid Interface Sci. 1988, 123, 523.

[2] G. H. Nancollas, T. F. Kazmierczak, E. Schuttringer, Corrosion 1981, 37, 76 .

[3] H. Cölfen, Macromol. Chem. Phys., Rapid Commun. 2001, 22, 219.

[4] M. Sedlak, M. Antonietti, H. Cölfen, Macromol. Chem. Phys. 1998, 199, 247.

[5] H. Cölfen, M. Antonietti, Langmuir 1998, 14, 582.

[6] J. M. Marentette, J. Norwig, E. Stockelmann, W. H. Meyer, G. Wegner; Adv. Mater. 1997, 9, 647.

[7] K. Kaluzynski, J. Pretula, G. Lapienis, M. Basko, Z. Bartczak, A. Dworak, S. Penczek, J. Polym. Sci., Part A: Polym. Chem. 2001, 39, 955.

[8] L. M. Qi, H. Cölfen, M. Antonietti, Angew. Chem. Int. Ed. 2000, 39, 604. 
[9] L. M. Qi, H. Cölfen, M. Antonietti, Chem. Mater. 2000, 12, 2392.

[10] H. Cölfen, L. M. Qi, Chem. Eur. J. 2001, 7, 106.

[11] M. Antonietti, M. Breulmann, C. G. Göltner, H. Cölfen, K. K. W. Wong, D. Walsh, S. Mann, Chem. Eur. J. 1998, 4, 2493.

[12] L. Qi, H. Cölfen, M. Antonietti, M. Li, J. D. Hopwood, A. J. Ashley, S. Mann, Chem. Eur. J. 2001, 7, 3526.

[13] Y. Kitano, K. Park, D. W. Hood, J. Geophys. Res. 1962, $67,4873$.
[14] S. Förster, E. Krämer, Macromolecules 1999, 32, 2783.

[15] S. Ramakrishnan, Macromolecules 1991, 24, 3753.

[16] L. Stryer, "Biochemistry", 4th edition, W. H. Freeman and Company, New York 1995, p. 590.

[17] C. S. Sikes, M. L. Yeung, A. P. Wheeler, "Inhibition of Calcium Carbonate and Phosphate Crystallization by Peptides Enriched in Aspartic acid and Phosphoserine", in: Surface Reactive Peptides and Polymers, C. S. Sikes, A. P. Wheeler, Eds., ACS, Washington 1991.

[18] D. Walsh, B. Lebeau, S. Mann, Adv. Mater. 1999, 11, 324. 